\title{
Efecto de la suplementación con una dosis masiva de vitamina $A$ en niños de 6 a 36 meses de edad
}

\author{
Alma E. Robles-Sardin, M. en C., ${ }^{(1)}$ Humberto Astiazarán-G arcía, M. en C., ${ }^{(1)}$ \\ Reyna Dávalos-N avarro, M. en C., (2) Luis Q uihui-Cota, M. en C.,1) \\ Rosa Ma. Cabrera-Pacheco, M. en C., (1) Mauro E. Valencia, Dr. en C. ${ }^{(1)}$
}

\begin{abstract}
Robles-Sardin A E, Astiazarán-García H, Dávalos-Navarro R, Quihui-Cota L, Cabrera-Pacheco RM, Valencia ME. Efecto de la suplementación con una dosis masiva de vitamina $A$ en niños de 6 a 36 meses de edad. Salud Publica Mex 1998;40:309-315.
\end{abstract}

\section{Resumen}

Objetivo. Evaluar el impacto de la suplementación con una dosis masiva de vitamina $A$ en el estado nutricio de dicha vitamina y del hierro, en niños de zonas urbanas mar ginadas de Hermosillo, Sonora, México. Material y métodos. Se seleccionaron 60 niños (6-36 meses), a los que se les administró una dosis de 100000 UI (6-12 meses) y 200000 UI (12-36 meses) de vitaminaA en el Día $N$ acional de Salud de la Secretaría de Salud (junio de 1994). Se midieron niveles séricos de retinol, carotenoides, hierro, transferrina y ferritina, en tres fases: basal, a las dos semanas y después de tres meses. Resultados. En la primera fase, la mediana de retinol fue $0.7 \mu \mathrm{mol} / \mathrm{l}$; en la segunda aumentó a $0.97 \mu \mathrm{mol} / \mathrm{l}(p<0.001)$, y en la tercera disminuyó a 0.83 umol/l ( $p>0.05)$. Un $6.3 \%$ de los niños presentó deficiencia severa de vitamina $A$ al inicio, y no se encontró ningún caso en las fases posteriores a la suplementación. En la fase basal, $42 \%$ de los casos presentaron deficiencia moderada y la mayoría fue normal en las siguientes fases. N o hubo diferencias en los niveles de hierro y transferrina ( $p>0.05)$, pero sí en ferritina $(p<0.001)$, lo que indica un mejoramiento en las reservas de hierro a los tres meses. Conclusiones. Las campañas de distribución de dosis masivas pueden tener un impacto importante en el estado nutricio de vitamina $A$ y hierro.

Palabras clave: deficiencia de vitamina A; estado nutricional; alimentación suplementaria; México

\author{
Robles-Sardin AE, Astiazarán-García H, \\ Dávalos-Navarro R, Quihui-Cota L, \\ Cabrera-Pacheco RM, Valencia ME. \\ Effect of masive vitamin A \\ supplement in children \\ from 3 to 36 months of age. \\ Salud Publica Mex 1998;40:309-315.
}

\begin{abstract}
A bstract
Objective. To evaluate the impact of a single massive dose of vitamin A on the vitamin and iron status in children of margined urban areas in Hermosillo, Sonora, Mexico. Material and methods. A total of 60 children received one dose of $100000 \mathrm{IU}$ (children from 6 to 12 months) and of $200000 \mathrm{IU}$ (children from 12 to 36 months) of retinol palmitate distributed by the $\mathrm{H}$ ealth Ministry in June, 1994. Serum levels of retinol, carotenoids, iron, transferrin and ferritin were measured at three periods: basal, after two weeks and after three months. Results. The mean for retinol basal values was $0.7 \mu \mathrm{mol} / \mathrm{l}$, after two weeks it was $0.97 \mu \mathrm{mol} / /(p<0.001)$ and after three months it was 0.83 $\mu \mathrm{mol} / \mathrm{l}(p>0.05)$. When basal values were measured, $6.3 \%$ of the children showed severe vitamin A deficiency, which disappeared in the periods following the supplement; additionally, $42 \%$ showed moderate deficiency which had decreased to less than $7 \%$ after three months. No differences were found for serum iron and transferrin levels $(p>0.05)$, however, ferritin levels increased $(p<0.001)$ after three months indicating an improvement in iron reserves. Conclusions. Massive dose distribution may have an important impact on vitamin A and iron status in children.
\end{abstract}

Key words: vitamin A deficiency; nutritional status; supplementary feeding; Mexico

(1) Centro de Investigación en Alimentación y Desarrollo,A.C. Hermosillo, Sonora, México.

(2) Secretaría de Salud Pública, Jurisdicción Sanitaria N 0.1, Hermosillo, Sonora, México.

Fecha de recibido: 11 de agosto de 1997 - Fecha de aprobado: 2 de abril de 1998

Solicitud de sobretiros: Dr. Mauro E. Valencia. Centro de Investigación en Alimentación y Desarrollo, A.C. Carretera a la Victoria, km. 0.6. AP 1735. Hermosillo, Sonora, México. 
L a deficiencia de vitamina A es un problema de salud pública de dimensiones mundiales. Lo anterior ha sido reconocido por el Grupo Consultor Internacional de Vitamina A en su reunión en Guatemala (IVACG). ${ }^{1}$ La deficiencia de vitamina A (DVA) es la principal causa de ceguera en los niños de países pobres. Por otra parte, desde la perspectiva subclínica, contribuye a aumentar significativamente la morbilidad y la mortalidad de los niños por infecciones comunes. ${ }^{2}$ Las anemias de origen nutricio, sobre todo las relacionadas con la deficiencia de hierro, son de gran importancia epidemiológica, junto con la DVA en las regiones pobres. El hecho de que las anemias persistan a pesar de que los consumos de hierro sean adecuados, refleja la posible contribución de otros factores ambientales en su etiología. ${ }^{3}$

En 1987 la Organización Mundial de la Salud (OMS) estimó que la DVA es endémica en 39 países, basándose en la prevalencia de signos clínicos oculares o de muy bajos niveles de vitamina A en sangre $(<0.35$ $\mu \mathrm{mol} / \mathrm{lo} 10 \mu \mathrm{g} / \mathrm{dl})$. Actualmente se estima que la DVA, incluyendo un nivel clínico y subclínico de grado severo o moderado, es un problema de salud pública en 60 países y que probablemente otros 13 están a punto de ingresar en esa categoría. Se ha estimado que de 2.8 a 3 millones de niños en edad prescolar están clínicamente afectados y que 251 millones más están en riesgo, en términos de salud y sobrevivencia. ${ }^{2}$

Ahora bien, desde el punto de vista de la salud pública en Latinoamérica, la deficiencia de vitamina $\mathrm{A}$ constituye un problema importante, principalmente en el niño. De acuerdo con la OMS, ${ }^{2}$ en Hermosillo, Sonora, 32\% de los niños de 2 a 7 años de edad tenían niveles de retinol sérico deficientes $(<0.70 \mu \mathrm{mol} / 1 \mathrm{o}$ $20 \mu \mathrm{g} / \mathrm{dl}$ ). Además, en una revisión de Rivera Dommarco y colaboradores, ${ }^{4}$ se muestran datos dietéticos que sugieren que en México existen deficiencias en el consumo de esta vitamina en algunos sectores de la población. México está considerado como parte de un grupo de países con deficiencia subclínica de vitamina A y con un problema significativo de salud pública. ${ }^{5}$

Con el objeto de combatir la deficiencia de vitamina A, así como sus efectos, se han puesto en marcha programas de salud en diferentes países tales como Guatemala, Indonesia y Tailandia, entre otros. ${ }^{6-8}$ Desde 1993 se está aplicando en México un programa consistente en la administración periódica de vitamina A a niños de 0.5 a 5 años de edad. El objetivo de este trabajo fue analizar el impacto sobre el estado nutricio de vitamina A y hierro de una dosis única de vitamina A (100 000 o 200000 UI) suministrada durante la campaña de la Secretaría de Salud Pública (junio de 1994), en un grupo de niños de Hermosillo, Sonora, México.

\section{Material y métodos}

Este estudio se llevó a cabo durante los meses de junio a septiembre de 1994. Las madres de los participantes fueron informadas acerca de los motivos del mismo y se indagó acerca de su interés en cooperar. En los casos en los que la madre dio respuesta afirmativa se obtuvo su consentimiento por escrito, según lo indica el protocolo que fue revisado y aprobado por el comité de ética de la institución, donde se realizó la evaluación (Centro de Investigación en Alimentación y Desarrollo, A.C.).

\section{Marco muestral}

Los niños seleccionados provenían de zonas urbanas marginadas de la ciudad de Hermosillo, Sonora, y además formaban parte del programa de suplementación del Día Nacional de Salud de la Secretaría de Salud Pública, que opera desde 1993. Por razones logísticas fue imposible obtener valores de peso y talla de los participantes. Los niños provenían de dos colonias consideradas de nivel socioeconómico bajo -El Apache y Lomas de Madrid-, de acuerdo con Camberos. ${ }^{9}$ Participaron 34 niños de 6 a 36 meses y 26 niñas de 6 a 35 meses de edad.

Los niños fueron seleccionados como parte de un procedimiento muestral intencional no probabilístico comparativo. Se escogieron 60 niños menores de tres años que recibieron la dosis de vitamina $\mathrm{A}(<1$ año= 100000 UI; $\geq 1$ año y $\leq 3$ años= 200000 UI), la cual es conocida como segura y efectiva. Se sabe que cuando la vitamina A es administrada en las dosis recomendadas raramente se presentan efectos adversos. ${ }^{10}$

\section{Procedimiento experimental}

Para el procedimiento de toma de muestra se seleccionó la punción con lanceta del dedo medio de la mano del niño, y consistió en la extracción de $1 \mathrm{ml}$ de sangre total sin anticoagulante. A cada niño se le tomaron tres muestras: una basal, una dos semanas después y otra a los tres meses. Una vez obtenida la muestra de sangre, se protegió de la luz, se etiquetó y se transportó bajo refrigeración $\left(4^{\circ} \mathrm{C}\right)$ en el menor tiempo posible al laboratorio donde se congeló a $-20^{\circ} \mathrm{C}$.

Para la determinación de vitamina A se empleó la técnica de inactivación con luz ultravioleta descrita originalmente por Bassey y colaboradores ${ }^{11}$ en 1946, y revisada y actualizada por el IVACG, ${ }^{12}$ la cual se basa en la detección de los dobles enlaces conjugados de las moléculas de retinol y carotenos a 328 y $460 \mathrm{~nm}$, respectivamente. El análisis se llevó a cabo en un espec- 
trofotómetro UV-Visible de doble haz (LAMBDA 3B UV-VIS Perkin Elmer).

El análisis de ferritina se hizo en suero por ensayo inmunorradiométrico basado en la detección de ferritina por medio de anticuerpos: un anticuerpo monoclonal antiferritina y un anticuerpo policlonal antiferritina con ${ }^{125}$ I marcado en fase líquida (Coat-ACount, Ferritin IRMA, Diagnostic Products Corporation, LosAngeles, California, EUA).

El hierro se determinó en suero; el fundamento de la prueba es que el hierro sérico es liberado de la transferrina (proteína transportadora de hierro) en presencia de ácido acético y es reducido a estado ferroso por la hidroxilamina y tioglicolato. Estos iones ferrosos forman inmediatamente un complejo con la ferrocina, que puede ser leído colorimétricamente. Las determinaciones se hicieron en un equipo automatizado SYNCHRON CX (Beckman Instruments Inc., 1993). La transferrina se analizó por un método nefelométrico automatizado ARRAY Systems que ha dado resultados específicos y reproducibles (Beckman Instruments Inc., 1995).

\section{Procedimientos estadísticos}

La normalidad de las distribuciones se analizó mediante las pruebas de: Kolmogorov-Smirnov, MartinezIglewicz y D'Agostino. Adicionalmente se realizó la prueba de Bartlett para analizar la homogeneidad de varianzas en cada uno de los tratamientos. El comportamiento de los desplazamientos de las distribuciones de vitamina A se analizó de acuerdo con lo sugerido por Flores y colaboradores ${ }^{13}$ en Brasil. Se llevó a cabo un análisis de varianza no paramétrico de un factor con la prueba de órdenes de Kruskal Wallis, bajo la hipótesis nula de igualdad de medianas y la prueba de comparaciones múltiples de Dunn. Todos los análisis se llevaron a cabo con los paquetes estadísticos NCSS, version 6.0.1 (Kaysville, Utah, EUA, 1995) y Graph Pad (Graph Pad Software INS., California, EUA).

\section{Resultados}

\section{Estado nutricio de vitamina A}

\section{Retinol sérico}

Las evaluaciones se iniciaron con 60 niños; se perdieron cuatro en la segunda fase y otros 22 a los tres meses por ausencia de respuesta o respuesta incompleta. En el cuadro I se muestran los valores de retinol sérico.
En la fase basal de la medición, la mediana de retinol sérico fue de $0.70 \mu \mathrm{mol} / 1(20 \mu \mathrm{g} / \mathrm{dl})$, lo cual indica que, de acuerdo con la clasificación de la OMS ${ }^{14}$ la mitad de los niños tienen bajos niveles de este indicador $(<0.70 \mu \mathrm{mol} / 1)$.

A las dos semanas de haber recibido la dosis, los niveles de retinol sérico aumentaron significativamente $(p<0.001) 0.26 \mu \mathrm{mol} / 1(7.6 \mu \mathrm{g} / \mathrm{dl})$, resultando en una mediana de $0.97 \mu \mathrm{mol} / \mathrm{l}(27.8 \mu \mathrm{g} / \mathrm{dl})$. Sin embargo, a los tres meses el valor disminuyó a 0.83 $\mu \mathrm{mol} / 1(23.8 \mu \mathrm{g} / \mathrm{dl})$ y no fue significativamente distinto del valor basal.

\section{Carotenoides}

Para este caso, a pesar de que la prueba de Bartlett no detectó heterogeneidad de varianzas, las distribuciones mostraron comportamientos anormales, por lo que se decidió aplicar la prueba de Kruskal Wallis y la prueba de Dunn para comparar las diferencias entre las medianas. Las diferencias en los niveles de carotenoides totales en suero, en las distintas fases de medición, también fueron significativas $(p<0.05)$. En el cuadro I se muestran los resultados que, si bien no reflejan el estado de nutrición de vitamina A, sí indican la ingestión reciente de estos precursores. ${ }^{15}$ Se considera que valores mayores a $40 \mu \mathrm{g} / \mathrm{dl}$ son adecuados, y entre 20 y $39.9 \mu \mathrm{g} / \mathrm{dl}$, marginales.

\section{Cuadro I}

INDICADORES BIOQUímICOS DEL ESTADO DE VITAMINA A $Y$ HIERRO EN NIÑOS DE 6 A 36 MESES EN LAS DIFERENTES fases del estudio. Hermosillo Sonora, 1994

\begin{tabular}{lccc} 
Indicador* & Basal & 2 semanas & 3 meses \\
Retinol $(\mu \mathrm{mol} / \mathrm{L})$ & $0.70^{\mathrm{a}}(60)$ & $0.97^{\mathrm{b}}(56)$ & $0.83^{\mathrm{a}}(34)$ \\
\hline & {$[5.0-54.4]$} & {$[13.9-67.0]$} & {$[18.9-32.9]$} \\
\hline Carotenoides $(\mu \mathrm{g} / \mathrm{dL})$ & $36.9^{\mathrm{a}}(60)$ & $40.4^{\mathrm{ac}}(54)$ & $47.9^{\mathrm{bc}}(34)$ \\
\hline & {$[17.2-81.6]$} & {$[17.28-65.3]$} & {$[25.9-69.12]$} \\
\hline Ferritina $(\mu \mathrm{g} / \mathrm{L})$ & $13.0^{\mathrm{a}}(56)$ & $10.8^{\mathrm{a}}(18)$ & $20.0^{\mathrm{b}}(41)$ \\
\hline & {$[1.7-60]$} & {$[4.5-27]$} & {$\left[6.8^{-}-70\right]$} \\
\hline Hierro sérico $(\mu \mathrm{g} / \mathrm{dL})$ & $78^{\mathrm{a}}(47)$ & $71^{\mathrm{a}}(18)$ & $69^{\mathrm{a}}(37)$ \\
\hline & {$[13-311]$} & {$[14-231]$} & {$[17-219]$} \\
\hline Transferrina $(\mathrm{g} / \mathrm{L})$ & $2.9^{\mathrm{a}}(41)$ & $3.0^{\mathrm{a}}(11)$ & $2.8^{\mathrm{a}}(33)$ \\
\hline & {$[3.5-3.9]$} & {$[2.3-3.4]$} & {$[2.3-4.1]$}
\end{tabular}

* Mediana, [ ] Rango, ( ) N o. de niños.

$\mathrm{N}$ ota: superíndices diferentes en el mismo renglón indican diferencia significativa $(p<0.05)$ 


\section{Estado nutricio de hierro}

Los resultados obtenidos en lo que respecta a niveles de ferritina se muestran en el cuadro I. En la fase basal, la mediana de ferritina sérica fue de $13 \mu \mathrm{g} / \mathrm{l}$, lo que los ubica ligeramente por arriba del nivel normal $(>12 \mu \mathrm{g} / \mathrm{l}) .^{3}$ En la fase de impacto en el corto plazo, es decir, después de dos semanas de haber recibido la dosis, los niveles no fueron diferentes a los iniciales $(p>0.05)$. Sin embargo, a los tres meses de la suplementación el valor de la mediana de ferritina aumentó a 20 $\mu \mathrm{g} / 1(p<0.001)$. Por otra parte, al analizar el porcentaje de la distribución de los niños con niveles bajos de ferritina sérica, o sea con un estado de reservas de hierro deficientes, se observó que $48 \%$ de los niños se ubicaban por debajo de $12 \mu \mathrm{g} / 1$ en la fase basal y que no mostraron cambios a las dos semanas. Sin embargo, a los tres meses el porcentaje de niños con reservas deficientes disminuyó a la mitad (figura 1). Cabe recordar que, aunque el nivel de vitamina A en la tercera etapa no fue diferente estadísticamente del nivel basal, sí se tuvo un mayor porcentaje de niños clasificados como normales.

\section{Discusión}

Los resultados muestran que para estos estados de deficiencia, los programas pueden tener un impacto favorable en las fases tempranas; sin embargo, después de tres meses tienden a retornar a su nivel inicial, es decir, a niveles de deficiencia moderada. Esto puede confirmarse al analizar el comportamiento de las tres distribuciones que se presentan en la figura 2. El primer desplazamiento ocurre hacia la derecha en la fase temprana posterior a la suplementación, lo que indica un aumento en los niveles séricos de la vitamina y por ende una mejoría en su estado nutricional. Sin embargo, a los tres meses se puede apreciar una tendencia de desplazamiento hacia su posición inicial, lo cual es congruente con el análisis discutido anteriormente.

Por otra parte, en la figura 3 se puede observar que en la etapa basal existían casos de deficiencia severa de la vitamina hasta en $6.6 \%$ (de los cuatro casos, tres pasaron a niveles normales después de dos semanas de la suplementación, y el otro alcanzó un nivel de $0.66 \mu \mathrm{mol} / 1-19 \mu \mathrm{g} / \mathrm{dl}-$, muy cercano a los niveles normales); asimismo, se presentaron casos de deficiencia moderada en $41.6 \%$. En la fase temprana posterior a la suplementación no se presentaron casos de deficiencia severa, y los casos de deficiencia moderada disminuyeron hasta el 7.1\%. A los tres meses de haber ingerido la dosis tampoco se observaron casos de deficiencia severa, y los de deficiencia moderada

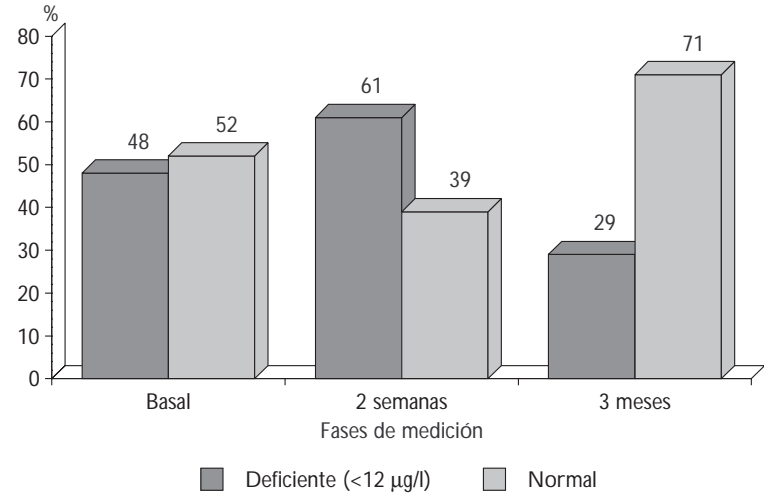

Figura 1. Distribución porcentual de los niveles DE FERRITINA SÉRICA EN LAS DIFERENTES FASES DE MEdición. Hermosillo, Sonora, 1994

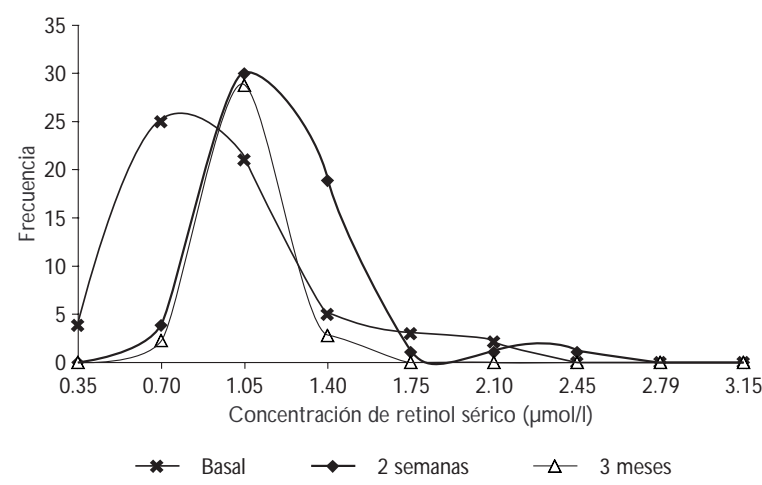

Figura 2. Distribución de frecuencias de retinol SÉrico en las fases de medición. Hermosillo, SonoRA, 1994

representaron sólo 5.9\%. Es importante hacer notar que no obstante que a los tres meses existían más niños clasificados como normales, muchos de ellos tendían a regresar a valores de retinol sérico muy cercanos a $0.70 \mu \mathrm{mol} / 1(20 \mu \mathrm{g} / \mathrm{dl})$. Esto indica la posibilidad de que sus niveles sigan descendiendo hasta llegar a la misma situación que presentaban en la fase basal.

Respecto a los carotenoides, los datos indican una mejoría desde el inicio del estudio; aunque a las dos semanas de la suplementación no hubo cambio ( $p>0.05)$, la ingestión a los tres meses sí se incrementó significativamente $(p<0.05)$; lo anterior puede reflejar variaciones estacionales con mayor disponibilidad de algunos productos vegetales.

Como se ha informado respecto a varias regiones del mundo, la ingesta de vitamina A puede fluctuar 


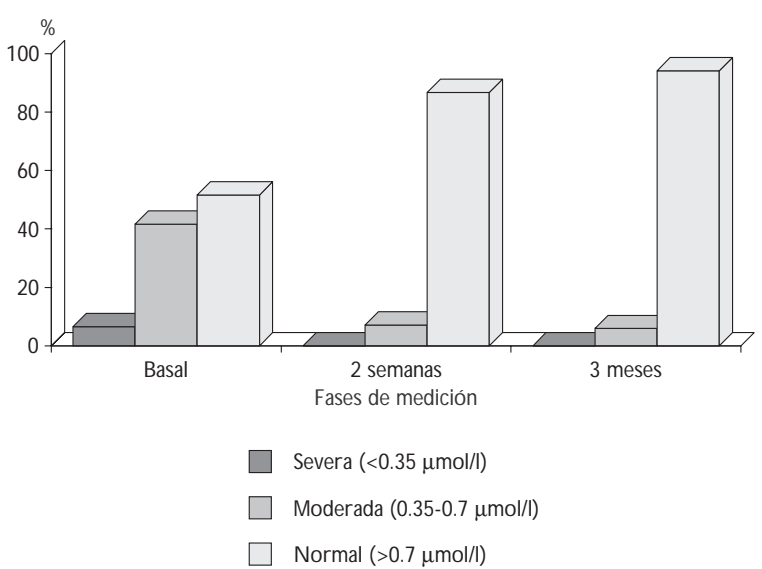

Figura 3. Distribución porcentual del estado de VITAMINA A (MEDIDA COMO NIVELES DE RETINOL SÉRICO). Hermosillo, Sonora, 1994

durante el año. ${ }^{16-18}$ Los datos dietarios de las regiones donde se han llevado a cabo estos estudios (sur y este de Asia), así como los de Estados Unidos de América, ${ }_{1}^{14}$ muestran que aproximadamente $50 \%$ de la vitamina A que se ingiere proviene de frutas y hortalizas. Ese tipo de alimentos usualmente se consume en mayor grado durante temporadas cortas. La etapa basal de este estudio inició en el mes de junio de 1994, lo que coincide con una mayor disponibilidad de frutas de temporada como mangos, papaya, duraznos, melón, etcétera, que pueden adquirirse, en algunos casos, a muy bajos precios, por lo que se puede presumir que la ingesta de vitamina A aumentó durante el estudio.

En otras regiones se ha administrado suplemento principalmente a sectores específicos de la población infantil. Uno de estos casos es Brasil, donde se administró suplemento con 200000 UI de vitamina A para 544 niños de áreas de bajos recursos. Los resultados fueron positivos, ya que la diferencia entre los niños con un estado adecuado de vitamina A y aquellos que no lo tenían desapareció después de la suplementación (30-45 días).

En otro estudio llevado a cabo por Bloem y colaboradores, ${ }^{8}$ se seleccionaron 134 niños entre 3 y 9 años de edad con signos clínicos (xerosis conjuntival) a los que se les dio suplemento de 200000 UI de vitamina A. El resultado que se obtuvo fue favorable, lo que se reflejó como una mejoría en los parámetros de retinol, proteína enlazante de retinol, hemoglobina, hematocrito, hierro sérico y saturación de transferrina, a las dos semanas de haber recibido la dosis.

En algunos estudios no se ha encontrado cambio en ferritina ${ }^{3,6}$ después de la suplementación con vita- mina A, pero en otros se ha observado un incremento en el nivel de ferritina a los cuatro meses de la suplementación de una dosis masiva. ${ }^{19}$ Este tipo de respuesta, en el presente estudio, puede atribuirse al aumento en la ingestión de hierro reflejada en el almacenamiento, ya que los niveles de hierro sérico se encontraban normales desde el inicio (cuadro I). Puede ser que al incrementarse la utilización metabólica del hierro (después de una suplementación), la respuesta fisiológica del organismo sea mejorar la absorción del hierro. ${ }^{3}$ Finalmente, es posible que al encontrarse niveles normales de hierro, el hierro absorbido haya aumentado las reservas, ya que uno de los factores que más influencia tienen en la deficiencia de absorción de hierro es la cantidad de depósitos en el organismo ${ }^{20}$ y, como se observa, los niveles de ferritina en las dos primeras fases del estudio están muy cerca del punto de corte de $12 \mu \mathrm{g} / \mathrm{l}$.

En lo que respecta al parámetro de hierro sérico, se observó que no hubo diferencia significativa entre las fases de medición (cuadro I). No obstante, es importante hacer notar que en todas las fases se clasifican como normales $(>50-170 \mu \mathrm{g} / \mathrm{dl}) .{ }^{20}$ En otros estudios se ha encontrado una mejoría en el estado de hierro sérico al administrar vitamina $\mathrm{A}^{8,19}$ en el corto y el mediano plazos (dos semanas, y dos y cuatro meses, respectivamente), aunque estos estudios se han hecho con poblaciones seriamente afectadas ya sea por deficiencia de hierro o de vitamina A. De igual manera, en el caso de transferrina sérica, se presentó un comportamiento similar al anterior (cuadro I).

Como se puede observar en los cuadros de resultados, se tuvieron distintos números de voluntarios en las distintas fases; hubo problemas particularmente en el muestreo después de dos semanas de haber administrado la suplementación. En varios casos no hubo voluntarios, ya que el muestreo coincidió con el periodo vacacional de los niños. Sin embargo, el mayor problema que se tuvo fue que la cantidad de muestra de sangre obtenida del niño resultó insuficiente para los análisis; lo anterior se debió a que inicialmente se tenía contemplado indagar sólo el estado de vitamina A después de la suplementación, pero se consideró importante explorar el estado de hierro en la medida de lo posible. En esa forma, es posible analizar la influencia que pueda tener la vitamina A en hierro en este tipo de población. En este estudio se tomaron como indicadores del estado de hierro: la ferritina, la transferrina y el hierro sérico.

De acuerdo con los resultados discutidos se puede apreciar que las campañas de distribución masiva pueden tener un impacto favorable para mejorar el estado de vitamina A en niños en riesgo. Para el desarrollo de 
esta investigación se utilizaron los criterios de clasificación de deficiencia de vitamina A de la OMS (1982). ${ }^{14}$ La mitad de la población de este estudio se encontraba por debajo de $0.70 \mu \mathrm{mol} / 1$, y $6.6 \%$, por debajo de 0.35 $\mu \mathrm{mol} / \mathrm{l}$. El desplazamiento de la distribución hacia la zona de seguridad, así como el hecho de que a los tres meses no había ningún niño en estado de deficiencia severa y que prácticamente el resto de los niños estaba dentro de los límites normales, lo confirma. Empero, también se observa la naturaleza transitoria de estas estrategias, pues los niveles del retinol tienden a regresar a su estado original.

Si bien puede pensarse que la distribución de dosis masivas es una estrategia de corto plazo, dependiendo de las circunstancias también puede ser de largo plazo. En 1992 se declaró a Indonesia como país libre de xeroftalmia, aunque aún persiste la deficiencia subclínica y es necesario seguir suministrando cápsulas. En algunas regiones de la India los programas de administración de megadosis han operado durante 20 años. Por lo tanto, en aquellas regiones o países en donde no se tengan estrategias alternativas es necesario mantener las que funcionen. Sin embargo, no hay que perder de vista otras estrategias que pueden ser de carácter regional o nacional, y que ofrezcan soluciones de mediano y largo plazos. ${ }^{1}$

De acuerdo con los resultados de este estudio, es conveniente que para niveles de deficiencia de este tipo las campañas se lleven a cabo cada seis meses, mientras no existan estrategias alternativas disponibles. Algunas de ellas serían la promoción de la lactancia materna, los programas de atención materno-infantil (incluyendo las inmunizaciones), la producción local o regional de alimentos ricos en vitamina $\mathrm{A}$, la educación para la salud y la nutrición. Otro tipo de opción es la fortificación de alimentos básicos, como alimentos de ablactación, alimentos en programas de asistencia y alimentos locales en las regiones problema. Estos programas pueden aplicarse en forma secuencial o en paralelo, buscando siempre las alternativas que funcionen en las regiones donde existen los problemas. Es necesario realizar más investigaciones sobre el contenido de carotenoides y su biodisponibilidad en diferentes regiones del país con acceso a distintas fuentes de estos alimentos en la dieta.

Adicionalmente se piensa que, cualquiera que sea la estrategia o estrategias seleccionadas por quienes toman las decisiones y por los gobiernos, es indispensable evaluar el impacto de dichos programas, de tal forma que se pueda conocer su funcionalidad, así como los cambios y la duración de las estrategias.
Por otro lado, con este estudio no se puede descartar la posibilidad de una influencia de la vitamina A en el estado nutrimental del hierro. Existe evidencia de un impacto positivo en regiones donde la deficiencia moderada de vitamina A y la anemia son endémicas; también hay investigaciones con animales, niños y personas de la tercera edad que han notificado un efecto igualmente positivo. ${ }^{6,21-24}$ Esos datos indican que el efecto principal de la vitamina A es mantener los niveles adecuados de hierro para proveer a los diferentes tejidos, incluyendo la médula espinal, con cantidades apropiadas de este mineral, y si bien en este estudio no se presentó un incremento en el nivel de hierro sérico, sí se observó un aumento en las reservas de hierro (ferritina).

Desde esa perspectiva, una intervención con suplementos de vitamina A en niños de países en vías de desarrollo puede proveer de seguridad, en términos de salud y sobrevivencia. Además, considerando que cada año millones de niños sufren las consecuencias fisiológicas de la deficiencia de vitamina A, es importante atender a esta asociación cuando se apliquen programas de nutrición y salud pública.

Es conveniente que se establezcan programas de educación para la salud para que las madres de familia tengan conocimiento de las consecuencias de la deficiencia no sólo de vitamina A, sino de otros nutrientes, cuyos efectos nocivos sobre la salud pueden evitarse de una manera sencilla.

Es importante que se sigan administrando dosis de vitamina A mientras el país no garantice otras posibilidades o estrategias que permitan a la población contar con un nivel de vida que ponga a su disposición una dieta variada, suficiente, sana y culturalmente aceptable por la población. En ese sentido el problema se reduce a lograr un desarrollo social y económico congruente que garantice a los pobladores de México alcanzar un nivel de vida adecuado.

\section{A gradecimientos}

Los autores agradecen al doctor Ernesto Rivera Claisse su valiosa colaboración y amplia disponibilidad durante el desarrollo de esta investigación. De igual forma, se agradece el invaluable apoyo del profesor Jorge Villarreal por las facilidades brindadas en las instalaciones del laboratorio de la Clínica La Salle para los análisis de ferritina. Asimismo se hace extensivo este agradecimiento a la química Gloria Jiménez Lizárraga, del Módulo de Atención Lomas de Madrid, por la ayuda brindada para la toma de muestras del estudio. 


\section{Referencias}

1. Grupo Consultor Internacional de laVitamina A.X VII IVACG meeting, $G$ uatemala.Virtual elimination of vitamin A deficiency: 0 bstacles and solutions for the year 2000;1996 marzo 18-22; Guatemala, Guatemala. 2. W orld Health 0 rganization. G lobal prevalence of vitamin A deficiency. MDIS W orking Paper 1995;(2):1-107.

3. Mejía LA, Chew F. Hematological effect of supplementing anemic children with vitamin A alone and in combination with iron. Am J Clin N utr 1988:48:595-600.

4. Rivera-D ommarco J, Hernández-Avila M, López-C ervantes M. Semana $\mathrm{N}$ acional de Salud, bases técnicas para la ministración de vitaminaA. Cuernavaca (Mor.): Instituto N acional de Salud Pública, 1993:1-11.

5. Mora J0. Vitamin A deficiency in Latin America and the Caribbean, 1994. N utriview 1994 Special Issue:1-5.

6. Mejía LA, Hodges RE,Arroyave C,Viteri F,Torun B.Vitamin A deficiency and anemia in Central A merican children. Am J C lin N utr 1977; 30:11751184.

7.Tanumihardjo SA, Muhilal,Yetty Yuniar, D ewi Permaesih, Zein Sulaiman, Darwin Karyadi et al.Vitamin A status in preschool age Indonesian children as assessed by the modified relative-dose-response assay. Am J Clin N utr 1990; 52:1068-1072.

8. Bloem MW, Wedel M, Van Agtmaal EJ, Speek AJ, Saowakontha A, Schreurs W HPO Vitamin A intervention: Short-term effects of a single, oral, massive dose on iron metabolism. Am J C lin N utr 1990; 50:332-338. 9. Camberos M. La fuerza de trabajo en una región, Sonora y el municipio de Hermosillo (tesis de maestría en Economía). México, D.F.: U niversidad N acional Autónoma de México, Facultad de Economía, 1984.

10. World Health 0 rganization. Vitamin A supplements. A guide to their use in the treatment and prevention of vitamin $A$ deficiency and xerophtalmia. Ginebra: W HO, 1988:10-12.

11. Bessey $O A$, Lowry $O H$, Brooks MJ, López JA. The determination of vitamin $A$ and carotene in small quantities of blood serum. J Biol Chem 1946;166:177.

12. G rupo Consultor Internacional de laVitaminaA. Biochemical methodology for the assessment of vitamin A status, a report of the International
Vitamin A Consultive Group. W ashington, D.C.: The N utrition Foundation, 1982.

13. Flores H, Azevedo MCNA, Campos FACS, Barreto-Lins MC, Cavalcanti AA, Salzano AC et al. Serum vitamin distribution curve for children aged 2-6 y known to have adequate vitamin $A$ status:A reference population. Am J C lin Nutr 1991; 54:707-711.

14. World Health $O$ rganization. Control of vitamin A deficiency and xerophthalmia. Report of a joint W HO /UN IC EF/USAID/H elen Keller International/IVACG meeting. Ginebra: W HO, Technical Report Series, núm. 672, 1982.

15. Gibson SR. Principles of nutritional assessment. Assessment of the status of vitamins A, D and E. N ueva York: 0 xford University Press 1990:377-412.

16. Moore T. Vitamin A. Amsterdam: Elsevier, 1957.

17.0 omen HAPC, Ten D oeschate J.The periodicy of xeroftalmia in South and East Asia. Ecol Food N utr 1973;2:207-217.

18. Sinha D P, Bang FB. Seasonal variation in signs of vitamin A deficiency in rural W est Bengal children. Lancet 1973;2:228.230.

19. Bloem MW, Wedel M, Egger R, Speek AJ, Schrijver, JAAP et al. Mild vitamin $A$ deficiency and risk of respiratory diseases and diarrhea in preschool and school children in Northeastern Thailand. Am J Epidemiol 1989; 131:332-339.

20. Bothwell T. $O$ verview and mechanisms of iron regulation. $\mathrm{N}$ utr Rev 1995;53:237-245.

21. Linder MC. N utrition and metabolism of vitamins. En: N utritional biochemistry and metabolism with clinical applications. 2a. edición. N ueva York: Elsevier, 1991:151-161.

22. Mejía LA, Hodges RE, Rucker RB. C linical signs of anemia vitamin $A$ deficient rats. Am J Clin N utr 1979; 32:1439-1444.

23. D onogghue S, Kronfeld DS, Berkowitz SJ, Copp RL. Vitamin A nutrition of the equine: Growth, serum biochemistry and hematology. J N utr 1981;111:365-374.

24. Wegner R, Ziegler B, Kruspl W, Syre B, Brubacher G, Pillat B. Bezienhungen zwischen dem vitaminstatus (vitamin $A, B 1, B 2$ und $C$ ), klinischen befunden un den ernährungsgewohnheiten in einer gruppe von alten leuten in W ien. W ien Klin W ochenschr 1979;91:557-562. En: Mejía LA.Vitamin A-nutrient interrelationships. Gainesville (Florida): Academic Press, 1986:69-100. 